\title{
Alternativa para recuperación de espacio público mediante infraestructuras verdes en Tunja, Colombia
}

\section{Alternative for the recovery of public space through green infrastructures in Tunja, Colombia}

Laura Estefanía Quintero González *

Recibido: 08 de agosto de 2017

Aceptado: 17 de noviembre de 2017

\section{Resumen}

La explosión demográfica global actual ha traído consigo el crecimiento desmedido de las ciudades, con áreas urbanas que se expanden, invadiendo zonas de importancia ambiental y, con ello, reduciendo la posibilidad de espacios públicos de calidad. Tunja es una de las ciudades en crecimiento de Colombia y su zona centro necesita una alternativa de recuperación y generación de espacios verdes públicos para evitar futuros fenómenos de densificación. La investigación tiene dos propósitos: determinar la disponibilidad del espacio público existente en el área urbana de la ciudad de Tunja mediante una caracterización y diagnóstico de disponibilidad; y, con ello, formular tipos específicos de infraestructuras verdes. Dadas las regulares condiciones de estado de la red de espacio público existente y la desigualdad en la conectividad espacial de los lugares dentro del sistema, se plantean tipos de infraestructuras vegetadas para su instalación en los lugares existentes y en los potencialmente nuevos.

Palabras clave: arquitectura verde, ciudades verdes, conectividad urbana, espacio público, gestión urbana.

\begin{abstract}
The current global demographic explosion has impacted in an excessive growth of cities, with urban areas invading key environmental places and with it, reducing the possibility of keeping good public spaces. Tunja is one of the growing cities of Colombia and its central zone needs an alternative of recovery and generation of public green spaces to avoid densification. The research proposes: firstly, to determine the availability of existing public space in the urban area of Tunja through a characterization and availability diagnosis; and, secondly, to formulate specific types of green infrastructure. By considering the regular state conditions of the existing public space network and the inequality in the spatial connectivity of the places within the system, a proposal of types of vegetated infrastructures is given to improve both existing and potentially places.

Keywords: green architecture, green cities, public space, urban connectivity, urban management.
\end{abstract}

\footnotetext{
* Filiación: Facultad de Ingeniería de la Universidad Pedagógica y Tecnológica de Colombia - AHCTTUM Quintero-G Ingeniería S.A.S. Correo electrónico: lauraestefania.quintero@uptc.edu.co

Proyecto de investigación titulado: Formulación de alternativas para recuperación de espacio público mediante infraestructuras verdes en Tunja, presentado como trabajo de grado para la obtención del título de Ingeniero Ambiental de la Facultad de Ingeniería de la Universidad Pedagógica y Tecnológica de Colombia.
}

Cómo citar: Quintero González, L. (2017). Alternativa para recuperación de espacio público mediante infraestructuras verdes en Tunja, Colombia. Revista de Urbanismo, 37, 1-17. http://dx.doi.org/10.5354/ru.v0i37.47058 


\section{Introducción}

La explosión demográfica global actual ha traído consigo el crecimiento desmedido de las ciudades, al mismo tiempo que las áreas urbanas se expanden, invadiendo zonas de importancia ambiental. Así mismo, con la urbanización y construcción de infraestructuras se reduce la posibilidad de tener grandes espacios públicos; además, de acuerdo con Alcântara Vasconcellos (2010):

Las personas quieren vivir en áreas residenciales atractivas y, al mismo tiempo, tener acceso a trabajo, a servicios, a áreas verdes... Estas posibilidades son negadas a aquellos sin acceso a automóvil... porque, aunque los automovilistas realicen viajes cortos... el dominio del tránsito representa su expropiación de espacios que antes eran agradables para andar a pie o en bicicleta y hoy son una actividad desagradable (p. 48).

Como se aprecia, la carencia de espacio público suficiente acarrea dificultades sociales entre las que se cuentan relaciones dispersas en las personas sujetas a una vida en las grandes ciudades, derivadas de un modelo desigual en cuanto a la accesibilidad al medio físico y espacio público (Lange Valdés, 2011), además de problemas del ambiente urbano como la interrupción de corrientes de aire y la falta de cobertura vegetal, aspectos que contribuyen a la formación de islas de calor, e incluso significan un detrimento en el patrimonio arquitectónico y paisajístico. Haciendo frente a esto, las estructuras verdes se muestran como una solución conveniente aplicable al problema de ausencia de espacio público suficiente, por lo que el presente artículo, como resultado de un proyecto de investigación (Quintero, 2017), pretende exponer una alternativa de recuperación de espacio público existente $y$ de generación de espacio público verde dentro del área de Conservación Histórica de la Ciudad de Tunja, capital del Departamento de Boyacá, ubicada en la región andina de Colombia.

Tunja posee un característico clima frío y una población en crecimiento que, de acuerdo con las proyecciones del Departamento Administrativo Nacional de Estadística, DANE, para el año 2020 tendrá un total de 206.827 habitantes en su territorio. La ciudad ha sido escogida como lugar objeto de estudio porque es una capital mediana en crecimiento económico, poblacional y de infraestructura urbana, y por su relevancia a nivel nacional como ciudad prehispánica y colonial con vocación cultural y estudiantil (Medina Roa, 2009).

La metodología planteada para el desarrollo de este trabajo está basada en el análisis de variables tales como las características físicas y usos del espacio público actual, la configuración espacial de la red de espacio público existente, la interconectividad entre lugares del espacio público actual y las características de los lugares potenciales para generar nuevo espacio público verde. Desde esta perspectiva, la metodología está encaminada a realizar un diagnóstico de la disponibilidad de espacio público en el área centro de la ciudad de Tunja, identificando los sitios que presentan condiciones favorables para la disposición de infraestructuras verdes; $y$, como resultado de ello, proponer de acuerdo con la localización y ubicación, tipos específicos de estructuras de arquitectura verde que más se ajusten a la disponibilidad y condiciones de los sitios identificados. El fin último del plan de trabajo diseñado es dar respuesta a la interrogante que enmarca esta investigación: ¿Se pueden formular alternativas de recuperación y generación de espacio público urbano a través de infraestructuras verdes en la ciudad de Tunja?, o lo que se resumiría en la aprobación o negación del planteamiento hipotético de si al determinarse la disponibilidad del espacio público existente en el área urbana de la ciudad de Tunja, es posible la formulación de tipos específicos de infraestructuras verdes para su recuperación y generación.

\section{Marco conceptual \\ El espacio público y la arquitectura verde}

Según Borja y Muxí (2000) "la historia de la ciudad es la de su espacio público" (p. 8), para lo cual definen el espacio público como el espacio principal del urbanismo, la cultura urbana y la ciudadanía, reflejado en la conformación de calles, plazas, parques y demás espacios de encuentro ciudadano, como resultado de la materialización de las relaciones entre habitantes y entre el poder y la ciudadanía.

Recientemente el concepto de sostenibilidad ha permeado la teoría y práctica de otras áreas relacionadas, derivando en conceptos particulares, como por ejemplo la arquitectura verde o también llamada ecodiseño, arquitectura ecológica o arquitectura del medio ambiente (U.S. Green Building Council, USGBC, 
2002). En todos estos casos este nuevo concepto comprende el diseño y la construcción de estructuras arquitectónicas basadas en principios ecológicos para el mejoramiento del ambiente social y ecológico de la zona en la que se instala un edificio o una estructura verde (Roy, 2008). Debido a su atractivo, el concepto ha generado el interés de entidades municipales por aumentar la cobertura vegetal, convirtiéndose actualmente en una política y prioridad en las ciudades (Pickett et al., 2011).

Así mismo, alrededor del mundo la arquitectura verde se ha implementado con diferentes propósitos, como en el proyecto de renovación urbana Ekostaden Augustenborg en Suecia que, en 2001, inauguró Augustenborg's Botanical Roof Garden, el primer jardín botánico de techo del mundo, instalación que abarca más de $9.000 \mathrm{~m}^{2}$ de tejados verdes, como respuesta a la necesidad de renovar este proyecto de vivienda y hacerlo nuevamente atractivo como en sus inicios (RolfsdotterJansson, 2009).

En Latinoamérica, particularmente en Ciudad de México (Suárez, Camarena, Herrera y Lot, 2001), se implementó infraestructura verde como una estrategia para crear corredores ecológicos en los pedregales en el sur de la ciudad y generar, de este modo, conectividad en la red de espacios públicos. De esta forma, mediante un modelo geodemográfico, se recopiló información para determinar la accesibilidad, los ecosistemas de pedregal, las áreas verdes disponibles $\mathrm{y}$ otros espacios que pudieran añadir características favorables al ecosistema. Dentro del estudio se identificaron los componentes clave del sistema de espacio público urbano; los nodos, espacios en los que confluyen elementos de la naturaleza y la actividad humana; las ligas, reflejadas en franjas vegetadas, parques lineales, corredores peatonales $y$ viales; y las redes, definidas como el conjunto de ligas y nodos.

En el contexto colombiano, la Secretaría del Medio Ambiente del Municipio de Medellín (2004-2007) realizó la formulación de los planes de manejo de las microcuencas la Bermejala, la Herrera, la Presidenta, la Malpaso, la India, la Ana Díaz y la Quintana, para convertirlas en siete parques lineales, instrumentos orientadores del territorio, que representarían 168.196 $\mathrm{m}^{2}$ de nuevo espacio público verde.
Ahora bien, dentro de las definiciones $y$ clasificaciones técnicas de lo que se entiende por arquitectura verde se enmarcan varios tipos de estructuras y edificaciones (Ragheb, El-Shimy y Ragheb, 2016), entre las que se puede mencionar las siguientes:

a) Los sistemas de aguas.

b) Las construcciones verdes, que son edificaciones hechas de materiales reciclados.

c) El diseño solar pasivo.

d) La arquitectura viva -tipología de interés para el desarrollo de esta investigación-, que abarca aquellas estructuras de arquitectura urbana que han tenido un proceso de disposición o instalación de algún tipo de cobertura vegetal.

\section{Infraestructuras de arquitectura viva}

En esta categoría de arquitectura es posible distinguir los siguientes aspectos:

- Techos verdes. Estructura producto de la instalación de vegetación en techos y terrazas de edificaciones, espacios generalmente inutilizados (Vandermeulen, Verspecht, Vermeire, Van Huylenbroec y Gellynck, 2011).

- Muros verdes. Definidos como la plantación y desarrollo de especies vegetales con algún objetivo, como ornamentación o producción de alimentos (Fernández-Cañero, Pérez, Quevedo, Pérez y Franco, 2008).

- $\quad$ Parques longitudinales. Espacios abiertos a lo largo de corredores naturales como riveras de ríos y canales, e incluso servidumbres abandonadas de trenes y aceras viales (Redondo Paredes, 2014). La denominación internacional es greenways, que en contextos metropolitanos surge como una herramienta para el uso sostenible de los diferentes espacios urbanos (Mayorga Mora, 2013).

- Bioswales o canales de filtración biológica. Canales de drenaje con vegetación que atenúan el potencial de inundaciones transportando el agua lluvia lejos de la infraestructura de alcantarillado crítica (Clark y Acomb, 2008). Estos sistemas de conducción de escorrentía de aguas pluviales proporcionan una ayuda a las alcantarillas, porque pueden absorber caudales bajos, o bien, filtrar los flujos de fuertes 
lluvias y tormentas (Natural Resources Conservation Service, 2005).

\section{Beneficios de la arquitectura viva}

A nivel mundial la arquitectura viva se ha convertido en una herramienta de mejoramiento de los espacios públicos urbanos, debido a sus múltiples beneficios ambientales entre los que se cuentan la mejoría del atractivo visual de las ciudades (Forest Research, 2010b), captura de contaminantes, reducción de niveles sonoros y tránsito de biodiversidad (Redondo Paredes, 2014). Así mismo, a nivel social proporciona mejoría en la salud mental de las personas con efectos reductores del estrés (Forest Research, 2010a) y la disminución de los índices de obesidad adulta (Ghimire et al., 2017); por último, a nivel económico genera ahorro en el mantenimiento de obras públicas (U.S. Environmental Protection Agency, EPA, 2015).

En general las infraestructuras verdes pueden contribuir tanto a la mitigación como a la adaptación al cambio climático, gracias a la multifuncionalidad de los espacios verdes (Vásquez, 2016); sin embargo, es importante enfatizar que estos beneficios en el entorno urbano, que claramente mejoran la calidad de vida, están orientados a disminuir los efectos y no las causas de problemas mayores, principalmente de tipo ecosistémico, que se desarrollan fuera de los límites urbanos, por lo que también es importante la planificación del ámbito urbano en relación con su entorno rural (Bolund y Hunhammar, 1999).

\section{Método}

\section{Caracterización y diagnóstico de disponibilidad}

Para dar cuenta de estos aspectos la investigación contempló dos instancias metodológicas:

Identificación del espacio público existente. Mediante documentos oficiales como el Plan de Ordenamiento Territorial de la ciudad, se reconocen aquellos lugares dentro de la zona de estudio que hacen parte de la red de espacio público, tales como parques, plazas y plazoletas, zonas verdes o casas-monumento.

Caracterización del espacio público existente. Esta descripción se hace desde la observación, en donde la inspección visual es una buena forma de establecer el buen o mal estado de los lugares, puesto que una de las perspectivas de la implementación de arquitectura verde en áreas urbanas es abordar el espacio público como un componente del paisaje urbano porque "además de funciones físicas, el espacio público configura el ámbito para el despliegue de la imaginación y la creatividad" (Pascual González y Peña Díaz, 2012, p. 27). A continuación, se muestran los seis aspectos a caracterizar en cada lugar:

1. Geometría del espacio. En cada uno de los sitios identificados se describen sus dimensiones en planta, las dimensiones de las fachadas, la cantidad y tipo de elementos presentes (ventanas, puertas, balcones, pórticos) y el material de los muros y los elementos, según el tipo de lugar descrito.

2. Mobiliario urbano/estado físico. Para el caso de zonas verdes, plazas o plazoletas, separadores y parques se hace un inventario y descripción de estado utilizando una escala (MB: Muy bueno, B: Bueno, R: Regular, M: Malo a MM: Muy malo), aplicada al mobiliario urbano presente (bancas, biciparqueaderos, casetas de ventas, cestas de basura, materas, protectores vegetales, elementos de comunicación, juegos para adultos e infantiles, luminarias y unidades de paradero de autobús), según Presidencia de la República de Colombia (1998). Por otra parte, a las casas-monumento, se les caracteriza el estado físico, compuesto por el estado de la fachada en general y de cada uno de los elementos, es decir, puertas, ventanas, balcones, pórticos, antejardines, y faroles presentes en ella.

3. Uso del espacio. A todos los sitios se les asigna uno o varios usos entre los que destacan las actividades deportivas, culturales, comercio informal o ventas ambulantes, lugar de paso o de acceso a otro lugar, contemplación paisajística y ocio, cultos religiosos, uso institucional $\mathrm{y} / \mathrm{o}$ en abandono, indicando la frecuencia en que se practican los usos listados (MF: Frecuente, F: Frecuente, PF: Poco Frecuente).

4. Seguridad y confort del sitio. Según Gómez y Moyano (2014), algunos aspectos que determinan la seguridad de un sitio son la iluminación nocturna, la seguridad frente al crimen de día, la seguridad frente al crimen de noche y la seguridad frente al tráfico. En cuanto al confort del lugar, se tienen en cuenta el aseo, la 
protección climática por parte de sombras arbóreas, el confort visual relacionado con la ausencia de contaminación $y$, el confort auditivo. Cada uno de estos ítems es calificado cualitativamente (MB: Muy bueno, B: Bueno, R: Regular, M: Malo, MM: Muy malo).

5. Localización y articulación estratégica. Se debe tener en cuenta el indicador de movilidad peatonal (deseo o disposición a caminar) el cuál revela la distancia que las personas están dispuestas a caminar por algún motivo de viaje. Así mismo, es importante aclarar que el valor de referencia por considerar al respecto no corresponde a la mayor distancia registrada que estuvo dispuesta a caminar alguna persona, sino a aquella distancia que la mayoría de la población está dispuesta a caminar. Luego de conocer este valor, se listan aquellos lugares que se encuentran a máxima dicha distancia del lugar que se está caracterizando. La razón de esta consideración es que el espacio público debe analizarse como una red que permita la articulación del tejido urbano, el desplazamiento y la comunicación de un punto a otro dentro de la ciudad (Pascual González y Peña Díaz, 2012).

6. Condiciones paisajísticas y atractivo visual. En esta instancia se realiza un inventario y descripción del estado físico (MB: Muy bueno, B: Bueno, R: Regular, M: Malo, MM: Muy malo) de las plantas ornamentales presentes (árboles, arbustos, césped, florales, trepadoras, acuáticas y epífitas). Por último, y como parte del atractivo visual, se caracteriza la señalización en el lugar que indique como mínimo su nombre, ya que es un elemento que aporta importancia, belleza y distinción (la descripción identifica los criterios de visible o poco visible y el estado en el que se encuentra (MB: Muy bueno, B: Bueno, R: Regular, M: Malo, MM: Muy malo).

\section{Valoración de niveles de importancia}

Consiste en la conversión de los valores cualitativos de la caracterización en valores cuantitativos, donde las categorías Muy bueno, Bueno, Regular, Malo y Muy malo tendrán valores equivalentes, que abracan de 5, 4, 3, 2, a 1 , respectivamente, para asignar a cada sitio un puntaje que sea indicador de aquellos lugares que necesitan mejora prioritaria.

Esta asignación cuantitativa se verifica de acuerdo con la matriz de valoración de importancia propuesta por Pinzón Botero, Torres y Colonia Manzano (2008), en el Estatuto del Espacio Público Municipio de Palmira Colombia donde cada aspecto (b. Mobiliario urbano/estado físico; c. Uso del espacio; d. Seguridad y confort; e. Localización y articulación estratégica; y f. Condiciones paisajísticas y atractivo visual) se valora de 1 a 3 , de tal forma que la sumatoria de los puntajes de los cinco aspectos sea el puntaje total del lugar. Sin embargo, para este estudio se han ampliado los rangos de evaluación de 1 a 5 (de 1 a 1,9 = Muy malo; de 2 a 2,9 = Malo; de 3 a 3,9 = Regular; de 4 a 4,9= Bueno y $5=$ Muy bueno).

Teniendo en cuenta que solo se valoran cinco aspectos de los seis caracterizados, el puntaje mínimo que puede obtener un determinado lugar de análisis es de 5 puntos, con un máximo de 25 puntos totales; para hacer visible el nivel de prioridad de recuperación de los sitios, se discriminan sus puntajes totales en categorías y códigos de color; además, se clasifican los lugares en las tipologías de espacio público gris o espacio público verde (Tabla 1), para así conocer aquellos sitios que carecen completamente de cobertura vegetal. Es de suma importancia aclarar que se caracterizan específicamente solo los aspectos (b), (c), (d), (e) y (f); el aspecto (a) (Geometría) no se toma en cuenta, puesto que las dimensiones y forma de un lugar no son un factor determinante del estado físico $y$, por ende, de una posible necesidad de recuperación; por el contrario, se puede llegar a sesgar información de prioridad de intervención en caso de que se valore la geometría, por ejemplo, si un lugar se valora como "bueno" por su gran tamaño es posible alterar el puntaje total del espacio, de tal forma que se ignoren algunas otras condiciones de mal estado que requieren una intervención apremiante. La geometría solo es caracterizada para poder tomar decisiones acerca del tipo, requerimientos y magnitud de la estructura verde que se desee implementar. 
Tabla 1

Matriz genérica de valoración de importancia de sitios

\section{Puntajes}

Lugares / Aspectos

$\begin{array}{llll}\text { (b) } & \text { (c) } & \text { (d) } & \text { (e) (f) }\end{array}$

Total

Valoración

Tipología

$\begin{array}{lc}\text { Nombre lugar } \mathrm{N}^{\circ} 1 & 5-9,9 \\ \text { Nombre lugar } \mathrm{N}^{\circ} 2 & 10-14,9 \\ \text { Nombre lugar } \mathrm{N}^{\circ} 3 & 15-19,9 \\ \text { Nombre lugar } \mathrm{N}^{\circ} 4 & 20-24,9 \\ \text { Nombre lugar } \mathrm{N}^{\circ} 5 & 25\end{array}$

Fuente: Adaptado de Estatuto del Espacio Público Municipio de Palmira - Colombia.

De esta forma, para el cálculo de los puntajes por cada lugar a ser intervenido, la metodología utilizada contempla las instancias que se describen a continuación.

\section{Procedimientos de cálculo del puntaje por aspecto}

Mobiliario urbano/Estado físico. Para el mobiliario urbano únicamente se toma en cuenta el promedio de las calificaciones del mobiliario existente en cada lugar, sin considerar la ausencia de algún otro tipo de equipamiento de espacio público; sin embargo, si un lugar de uso público carece de todo tipo de mobiliario urbano, se le asigna la calificación más baja en este aspecto. Ahora bien, para el caso del estado físico, la valoración será igual al promedio de las calificaciones de los dos ítems que componen este aspecto: el estado físico general de las fachadas y el estado físico de los elementos de las fachadas, donde cada ítem se cuantifica haciendo el promedio de las calificaciones que el mismo contenga.

Uso del espacio. Su valoración es la relación entre los usos ideales de un sitio y los usos reales del mismo (Tabla 2), para lo que se proponen como usos ideales: las actividades deportivas y culturales, ser lugares de acceso a otros, la contemplación paisajística, el uso institucional y/o comercial y los cultos religiosos, en frecuencias que abarcan desde muy frecuente y frecuente; y como usos no ideales, estar en abandono y las ventas ambulantes en cualquier frecuencia.

Tabla 2

Valoración uso del espacio

\begin{tabular}{|c|c|c|c|}
\hline \multicolumn{3}{|c|}{ Relación uso-frecuencia ideales con uso-frecuencia reales } & \multirow{2}{*}{ Valoración } \\
\hline № Usos ideales & Frecuencias ideales & № Usos no ideales & \\
\hline Dos o más & En todos los usos & Ninguno & 5,0 \\
\hline Dos o más & En algunos usos o en ningún uso & Ninguno & 4,5 \\
\hline Uno & Ninguna & Ninguno & 3,5 \\
\hline Dos o más & En todos los usos & Uno o más & 3,0 \\
\hline Uno & Ninguna & Uno o más & 1,5 \\
\hline Ninguno & Ninguna & Uno o más & 1,0 \\
\hline
\end{tabular}

Fuente: Elaboración propia. 
Seguridad y confort del sitio. Para obtener la valoración de este aspecto basta con tomar los valores equivalentes y hacer el promedio de las calificaciones del listado de ítems caracterizados.

Localización y articulación estratégica. Inicialmente, se realiza una agrupación en intervalos de clase de los datos recolectados (número de lugares cercanos que tenga cada sitio); el número de intervalos por contemplar es de cinco, que corresponde al número de niveles de valoración del aspecto. Teniendo en cuenta esto, se calculan el rango y la amplitud de los intervalos para construir una tabla de equivalencia, en la que se relaciona el número de sitios cercanos con el puntaje de valoración del aspecto. En caso de que para cinco intervalos la amplitud sea mayor que ocho, se debe ampliar el espectro de valoración para ser más específico en la asignación de los puntajes, y entonces el nuevo número de intervalos a tomar será de 41 , en donde los puntajes del aspecto irán de 1,0 hasta 5,0, aumentando una décima de intervalo a intervalo, de manera tal que los primeros diez intervalos $(1,0-1,9)$ tienen una valoración de Muy malo, los siguientes diez $(2,0-2,9)$ son valorados como Malo, los siguientes diez $(3,0-3,9)$ obtienen una valoración de Regular, los siguientes diez $(4,0-4,9)$ se les asigna una valoración de Bueno y el intervalo número 41 con un puntaje de 5,0 es entonces el único con la valoración de Muy bueno.

Condiciones paisajísticas y atractivo visual. La valoración de este aspecto constituye el promedio de los puntajes equivalentes de los tres ítems que lo componen: el estado de las plántulas; el de los árboles y el de la señalización o distinción (para el caso de las casas monumento, solo se promedia el primer y tercer ítem, ya que son lugares en los que no hay presencia arbórea). Para la valoración del primer ítem, solo se promedian los puntajes equivalentes de las plántulas existentes en el lugar. El segundo ítem, estado de los árboles, está condicionado por su presencia, para lo cual se toma como indicador de presencia el ítem "protección climática (sombra arbórea)" del aspecto seguridad y confort, debido a que relaciona si la cantidad de árboles registrados son suficientes o no para aportar confort en el espacio caracterizado. Así pues, la calificación total de este segundo ítem será el promedio de la calificación de estado de los árboles y la sombra arbórea del lugar. La calificación del tercer ítem, estado de la señalización o distinción, equivale al estado físico de la señalización que pueda tener el lugar.

\section{Categorización de los componentes del espacio público}

Pinzón et al. (2008), proponen dos categorías para determinar los componentes del espacio público: aquellos sitios que conforman grupos y aquellos lugares que se encuentran aislados dentro de la distribución espacial de la red de espacio público. Esta distinción permite conocer si la red está muy bien interconectada, o por el contrario, fragmentada. Para este estudio, la categorización se logró mediante una relación directa con la valoración del aspecto localización y articulación estratégica (Tabla 3).

Tabla 3

Categorización de los componentes

\begin{tabular}{c|c}
\hline Localización y articulación estratégica & Categorización \\
\hline $1-1,9$ (Muy malo) & Lugares o pequeños grupos de lugares aislados \\
\hline $2-2,9$ (Malo) & Lugares medianamente agrupados \\
\hline $3-3,9$ (Regular) & Lugares mayormente agrupados \\
\hline 5 (Muy bueno) &
\end{tabular}

Fuente: Elaboración propia. 
Identificación, caracterización y selección de sitios potenciales

Finalmente, se consideran sitios potenciales para la generación de espacio público verde los terrenos baldíos, corredores viales, corredores hídricos, superficies de muros en vía pública, amplios andenes y antejardines. Luego de identificar aquellos sitios potenciales, deben describirse sus características físicas, de uso actual, de uso del suelo, seguridad y confort, y presencia vegetal, con el fin de conocer las condiciones de la posible instalación de algún tipo de infraestructura verde. Finalmente, se realiza una selección de sitios potenciales que sean principales candidatos, de acuerdo con la configuración espacial de la red de espacio público (espacios públicos agrupados y espacios públicos aislados), con el propósito de generar nuevos nodos y ligas de interconexión entre los espacios aislados y los grupos de espacios y viceversa.

\section{Resultados}

\section{Caracterización del área de estudio}

Este estudio se ha centrado en analizar los componentes del espacio público de la zona centro de Tunja, puesto que es una de las que presenta mayores motivos de desplazamiento de las personas desde y hacia ella y, por ende, mayor necesidad de espacio público en buenas condiciones. De este modo, se escoge como área de estudio la Zona de Conservación Histórica de Tunja Código ZUCH - Subzona urbana IV (Mapa de Zonificación Urbana División Política Administrativa Urbana del Plan de Ordenamiento Territorial de la ciudad de Tunja, 20002009).

\section{Descripción del estado actual del espacio público existente en el área de estudio y diagnóstico de disponibilidad}

Identificación del espacio público existente. Se identifican alrededor de cien lugares de uso público (plazas, plazoletas, parques, zonas verdes, separadores y monumentos o casas históricas (Figura 1), los cuales en su mayoría son espacios públicos grises -71 exactamente- y son relativamente pocos los espacios públicos verdes; además, 66 de los 71 lugares de espacio público gris son casas-monumento.

Caracterización del espacio público existente. La gran mayoría de los sitios cuenta con luminarias en buen estado, las cuales son un elemento mínimo que debe tener cualquier espacio público; los lugares con mayor y mejor amueblamiento son los parques, pues en su mayoría cuentan con bancas, cestas de basura, elementos de comunicación y juegos para adultos $y$ niños. Por otra parte, el estado físico general de las fachadas de los lugares catalogados como monumentos es Regular; aunque hay algunos monumentos de estado Bueno o Muy bueno, que coinciden con un uso institucional o comercial.

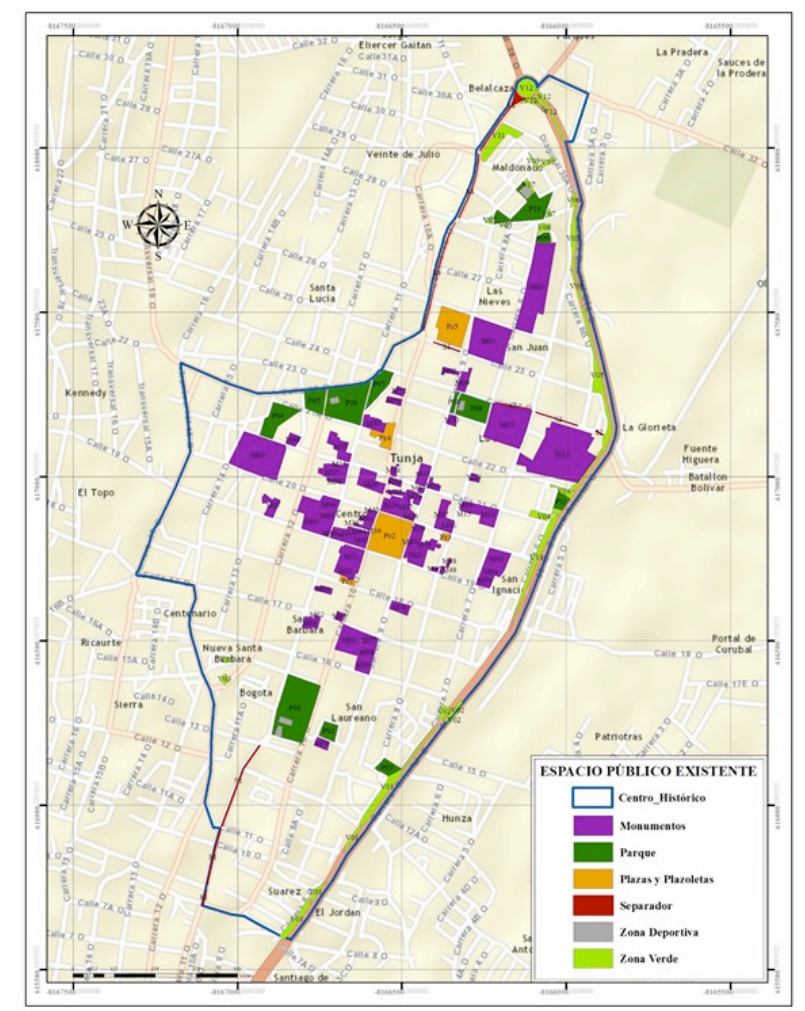

Figura 1. Espacio público existente en la zona de conservación histórica de Tunja.

Fuente: Elaboración propia.

En la caracterización del uso del espacio, se evidencia que dentro de la red de espacio público existen dos clases de espacios: la primera encierra los tipos de lugares en los que la tendencia de uso es como medio físico de acceso a otros sitios, o bien, como escenarios de desplazamiento a pie y, no son lugares de destino con un propósito específico y previamente planificado; dentro 
de esta clase están las zonas verdes y los separadores. La segunda clase incluye lugares a los cuales las personas se dirigen con un propósito o tarea específicos (trámites, acceso a educación, actividades religiosas, esparcimiento y deporte), y no por azar o necesidad de acceso a otros lugares; este es el caso de los parques y los monumentos. En el caso de las plazas y plazoletas sucede que la mayor parte del tiempo pertenecen a la primera clase y, ocasionalmente, son parte de la segunda clase, esto cuando se realiza algún tipo de evento cultural o comercial. Dicho esto, aquellos lugares de la primera clase, en los que el flujo y desplazamiento de personas es continuo y el tiempo de permanencia es corto, son catalogados como espacios conectores; $y$, aquellos lugares de la segunda clase, en los que convergen elementos de la naturaleza y actividades humanas (Suárez et al., 2011), son catalogados como nodos; así mismo, las plazas y plazoletas tienen una doble función, ser conectores o nodos, según el uso que se les asigne.

La seguridad frente al crimen, en general se percibe de Regular a Buena, aunque en los lugares más aislados de la red se percibe de Regular a Mala. Luego de comparar los datos de iluminación nocturna y seguridad frente al crimen, en la noche se observa que en general los lugares con una iluminación nocturna Buena o Muy buena tienen seguridad nocturna Regular y lugares con Regular iluminación nocturna tienen Muy mala seguridad nocturna, aunque también se registran lugares que con
Buena iluminación tienen Muy mala seguridad nocturna. Esto indica que, aunque la iluminación nocturna es un factor importante para la seguridad de un lugar, no es determinante para catalogarlo como completamente seguro. La zona más segura frente al tráfico dentro de la red de espacio público es su zona centro, calificada como Muy buena, ya que es el área peatonal por excelencia.

Para caracterizar la localización y articulación estratégica, el criterio de cercanía (distancia con mayor deseo de viaje) es entre $500 \mathrm{~m}$ y $600 \mathrm{~m}$ (cinco a seis cuadras de $100 \mathrm{~m}$ ), para lo cual la distancia escogida fue la mínima, esto es $500 \mathrm{~m}$. La información recolectada se contextualiza en un mapa que permite visualizar el número de lugares cercanos que tiene cada espacio público y que permite dar paso a la categorización de los componentes del espacio público (Figura 2).

Valoración de niveles de importancia. Luego de calcular los puntajes de cada lugar, se logra obtener un listado de lugares que son prioridad para recuperar (sitios con valoraciones de Bajo o Muy Bajo, como se muestra en la Tabla 4). Sin embargo, puede afirmarse que toda la red de espacio público de la Zona de Conservación Histórica de la ciudad de Tunja es susceptible de mejorar e intervenir, ya que en promedio la valoración de la red completa es de 15,67 puntos, es decir un nivel Medio, teniendo en cuenta además que ningún sitio de la red tiene una valoración Alta o Muy Alta. 
Tabla 4

Propuesta de recuperación de espacio público existente

\begin{tabular}{|c|c|c|}
\hline Lugar & \multirow{13}{*}{ 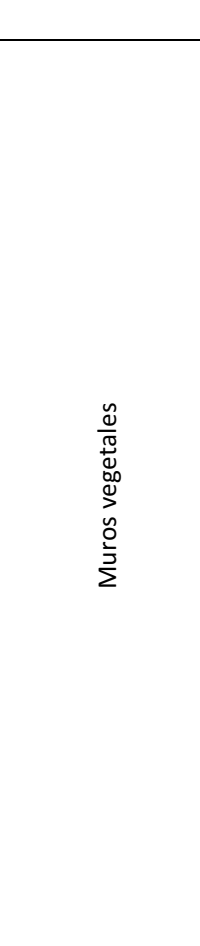 } & Descripción \\
\hline $\begin{array}{l}\text { Casa Familia } \\
\text { Domínguez }\end{array}$ & & \multirow{12}{*}{$\begin{array}{l}\text { Se propone un jardín vertical, que realce los elementos más importantes de estas edificaciones, que para e } \\
\text { caso son los pórticos, entonces, el jardín vertical estaría dispuesto en forma de arco alrededor del pórticc } \\
\text { existente, observándose un doble pórtico, uno de piedra en el interior y otro de vegetación en el exterior. Es } \\
\text { importante mencionar que el ancho del jardín vertical no debe superar el ancho del pórtico, para que se vea } \\
\text { proporcional y no le reste importancia a este elemento como parte del patrimonio arquitectónico. De igua } \\
\text { manera, se puede complementar esta intervención con la instalación de macetas con plantas florales en los } \\
\text { balcones de las casas que los posean. }\end{array}$} \\
\hline Casa Eduardo Santos & & \\
\hline $\begin{array}{l}\text { Casa Comunidad } \\
\text { Religiosa - Familia } \\
\text { Segura }\end{array}$ & & \\
\hline $\begin{array}{l}\text { Casa Don Juan de } \\
\text { Castellanos } \\
\text { (Historiador) }\end{array}$ & & \\
\hline $\begin{array}{l}\text { Gimnasio Gran } \\
\text { Colombiano }\end{array}$ & & \\
\hline $\begin{array}{c}\text { Casa Diagonal Legión } \\
\text { de María }\end{array}$ & & \\
\hline Casa Juan de Pineda & & \\
\hline $\begin{array}{c}\text { Casa Carrera } 10 \text { N. } \\
17-11\end{array}$ & & \\
\hline $\begin{array}{l}\text { Casa Enrique Medina } \\
\text { Flores }\end{array}$ & & \\
\hline $\begin{array}{c}\text { Casa Carrera } 10 \text { N. } \\
21-20\end{array}$ & & \\
\hline $\begin{array}{c}\text { Casa Carrera } 9 \text { N.o 17- } \\
98\end{array}$ & & \\
\hline $\begin{array}{l}\text { Universidad Juan de } \\
\text { Castellanos Centro }\end{array}$ & & \\
\hline $\begin{array}{l}\text { Lote Baldío (Esquina } \\
\text { Calle } 21 \text { con Carrera } \\
\text { 7) }\end{array}$ & $\begin{array}{c}\text { Parque/Terrazas } \\
\text { y jardines }\end{array}$ & $\begin{array}{l}\text { Se aconseja la instalación de un parque y, para dar atractivo visual, la instalación de jardines y plantas florales. } \\
\text { Para el servicio de las personas, es necesaria también la instalación de mobiliario urbano, como luminarias, } \\
\text { bancas, cestas de basura y juegos para adultos o para niños. Por último, dada la pendiente del terreno, el } \\
\text { diseño a manera de terrazas o niveles es una buena opción. }\end{array}$ \\
\hline $\begin{array}{l}\text { Zonas verdes 1-2-3 } \\
\text { Av. Oriental }\end{array}$ & \multirow{2}{*}{$\begin{array}{l}\text { Ajardinamiento } \\
\text { de fachada o } \\
\text { ajardinamiento } \\
\quad \text { vertical }\end{array}$} & \multirow{2}{*}{$\begin{array}{l}\text { Estas zonas verdes tienen una pronunciada pendiente de terreno que se debe al diseño vial de la Avenida } \\
\text { Oriental, puesto que son producto de los cortes laterales o taludes del corredor vial; por esta misma razón, se } \\
\text { recomienda la instalación de un ajardinamiento vertical. }\end{array}$} \\
\hline $\begin{array}{c}\text { Zonas verdes } 3 \text { Barrio } \\
\text { Lidueña }\end{array}$ & & \\
\hline $\begin{array}{c}\text { Zona verde Glorieta } \\
\text { Norte }\end{array}$ & $\begin{array}{l}\text { Jardines/ } \\
\text { bioswales/ } \\
\text { muro vegetal }\end{array}$ & $\begin{array}{l}\text { Se recomienda la instalación de bioswales y plantas florales. Además, para la recuperación del monumento que } \\
\text { se encuentra en uno de los separadores que conforman esta zona verde, se propone la instalación de un muro } \\
\text { vegetal dispuesto detrás de dicho monumento, lo suficientemente alto e iluminado en las noches para que lo } \\
\text { resalte. }\end{array}$ \\
\hline $\begin{array}{l}\text { Zonas verdes 1-2 } \\
\text { Barrio Santa Bárbara }\end{array}$ & $\begin{array}{l}\text { Parque/ } \\
\text { Jardines }\end{array}$ & $\begin{array}{l}\text { Se da la prioridad de recuperación porque una de las zonas corresponde a un lote baldío para el que se } \\
\text { propone la instalación de un parque con su respectivo mobiliario urbano y jardines de plantas florales. }\end{array}$ \\
\hline Parque Barrio Lidueña & $\begin{array}{l}\text { Jardines/ } \\
\text { muro verde }\end{array}$ & \multirow{2}{*}{$\begin{array}{l}\text { Debido a que estas dos zonas están contiguas al parque, se recomienda ampliar dicho parque para incluirlas, de } \\
\text { modo que se instalen jardines y algunos bioswales contiguos a la vía. Además, en dicha ampliación se debe } \\
\text { instalar el mobiliario urbano necesario como bancas, cestas de basuras y luminarias. }\end{array}$} \\
\hline $\begin{array}{l}\text { Zonas verdes 1-2 } \\
\text { Barrio Lidueña }\end{array}$ & $\begin{array}{l}\text { Jardines/ } \\
\text { bioswales }\end{array}$ & \\
\hline $\begin{array}{l}\text { Zona verde } 5 \text { Barrio } \\
\quad \text { Maldonado }\end{array}$ & $\begin{array}{l}\text { Jardines/ } \\
\text { bioswales }\end{array}$ & $\begin{array}{l}\text { Se propone la instalación de unas escalinatas, ya que es un terreno en pendiente, de paso y acceso a las } \\
\text { viviendas aledañas. Adicionalmente se propone la instalación de jardines y plantas florales alrededor de las } \\
\text { escalinatas. }\end{array}$ \\
\hline $\begin{array}{l}\text { Plaza de los Muiscas } \\
\text { (Nieves) }\end{array}$ & Bioswales & $\begin{array}{l}\text { Se recomienda el reemplazo del mobiliario urbano, principalmente de las bancas. Además, se recomienda la } \\
\text { instalación de bioswales en los andenes alrededor de la plaza, puesto que al interior de esta se realizan } \\
\text { actividades culturales y comerciales periódicamente, así que la intervención se propone en su contorno. }\end{array}$ \\
\hline $\begin{array}{l}\text { Zonas verdes } \\
\text { intersección Terminal } \\
\text { de Transportes }\end{array}$ & $\begin{array}{l}\text { Jardines/ } \\
\text { Bioswales }\end{array}$ & Se recomienda la instalación de bioswales y plantas florales. \\
\hline $\begin{array}{l}\text { Zonas verdes 1-2-3-4 } \\
\text { Barrio Maldonado } \\
\text { (Parque) }\end{array}$ & Bioswales & $\begin{array}{l}\text { Se propone la instalación de bioswales, ya que gracias a su ubicación entre vías pueden conformar buenos } \\
\text { sumideros biológicos. }\end{array}$ \\
\hline
\end{tabular}




\begin{tabular}{|c|c|c|}
\hline $\begin{array}{l}\text { Parque Barrio } \\
\text { Maldonado }\end{array}$ & $\begin{array}{l}\text { Ajardinamiento } \\
\text { de fachada o } \\
\text { ajardinamiento } \\
\quad \text { vertical }\end{array}$ & $\begin{array}{l}\text { Dado que el terreno en su parte alta es considerablemente llano y luego, hacia la Carrera } 8 \text { con Calle } 29 \text { el } \\
\text { terreno es bastante inclinado se recomienda, en la zona inclinada, la instalación de un ajardinamiento vertical, } \\
\text { además de la poda de varios arbustos para que se amplíe la visibilidad en el parque y, por ende, la seguridad } \\
\text { del lugar. Adicionalmente, para otorgarle distinción al espacio, se propone en las entradas y accesos principales } \\
\text { al parque la instalación de un arco vegetal (variación del muro vegetal), el cual se constituye por una estructura } \\
\text { metálica en forma de arco recubierta por plantas trepadoras o epífitas. }\end{array}$ \\
\hline Parque de las Aguas & $\begin{array}{l}\text { Jardines/ } \\
\text { Bioswales }\end{array}$ & $\begin{array}{l}\text { Se recomienda la reactivación de las fuentes de agua y ajardinar con plantas florales, además, gracias a la } \\
\text { amplitud del andén se propone la instalación de bioswales que enmarquen el área del parque. }\end{array}$ \\
\hline $\begin{array}{l}\text { Monumento al } \\
\text { Ajedrez }\end{array}$ & Bioswales & $\begin{array}{l}\text { Se propone la instalación de una cerca vegetal con su respectivo protector vegetal; esta cerca debe ser a una } \\
\text { altura media, en la que se permita la visualización del monumento, pero se impida en alguna medida el acceso } \\
\text { a él para evitar su deterioro (Figura } 4 \text {.). }\end{array}$ \\
\hline Separador Carrera 11 & Arcos vegetales & $\begin{array}{l}\text { Se propone una serie de arcos vegetales a lo largo de este separador vial que contiene en sí una ciclovía, para } \\
\text { aislar a los ciclistas del tráfico vehicular y, a su vez, generar una barrera acústica entre los flujos de la carrera. } \\
\text { Finalmente, con los arcos terminados se observará un túnel verde con gran atractivo visual desde fuera y } \\
\text { dentro de la ciclovía. }\end{array}$ \\
\hline
\end{tabular}

Fuente: Elaboración propia.

Categorización de los componentes del espacio público. Esta categorización de los lugares permite ver que los lugares agrupados tienen una única ubicación, es decir, hay un único grupo de lugares catalogados como agrupados y se encuentra aproximadamente en el centro del área de estudio. Además, alrededor de los lugares agrupados se encuentran aquellos medianamente agrupados, formando un anillo que a su vez se encuentra rodeado por los lugares más alejados de la red ubicados en la periferia de la zona de estudio. Entonces, la configuración espacial de la red de espacio público es concéntrica, en donde la mayor cantidad de lugares se encuentra en su centro espacial $y$, a medida que se avanza hacia el perímetro de la zona, la cantidad de lugares públicos disminuye (Figura 2).

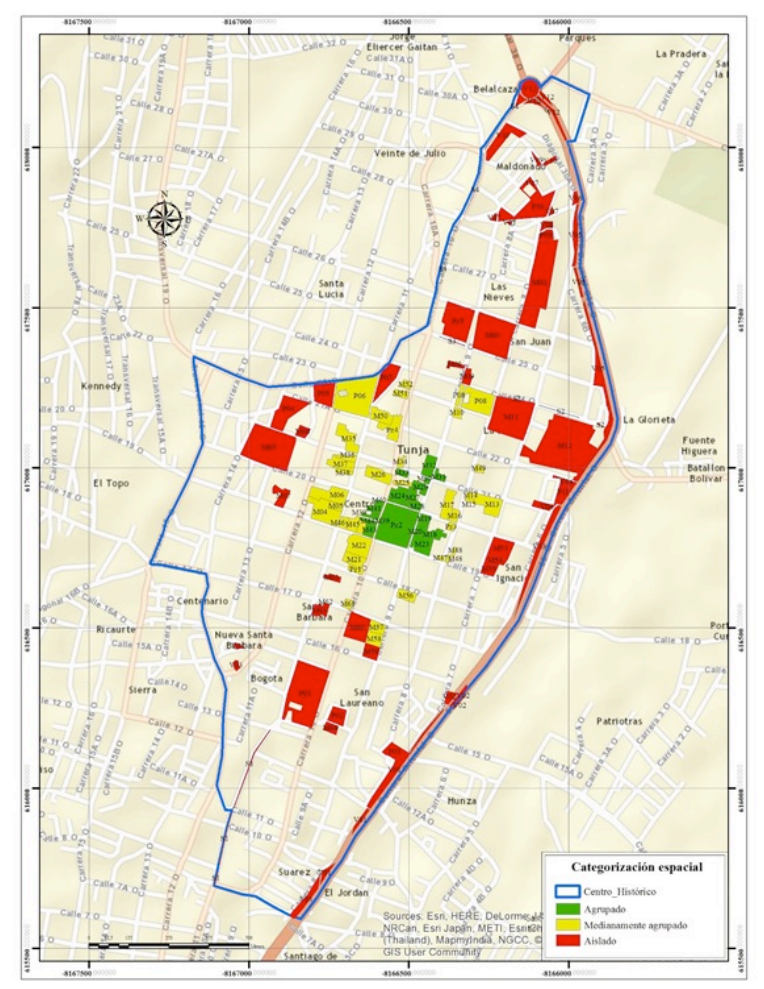

Figura 2. Configuración espacial de la red de espacio público existente.

Fuente: Elaboración propia.

Identificación de sitios potenciales. Dentro de los sitios identificados como potenciales de constituirse en un nuevo espacio público verde se identifican lotes baldíos, muros, antejardines y varias vías, los cuales fueron identificados y seleccionados en las cercanías de la periferia de la zona de estudio, puesto que es la parte que cuenta con menos lugares públicos y necesita fortalecerse la conectividad. En la Figura 3 se puede observar de manera gráfica y más detallada el criterio de selección de los lugares potenciales, es decir, la 
configuración espacial de la red y, además, se visualiza en dónde se ubican dichos lugares dentro de la zona de estudio.

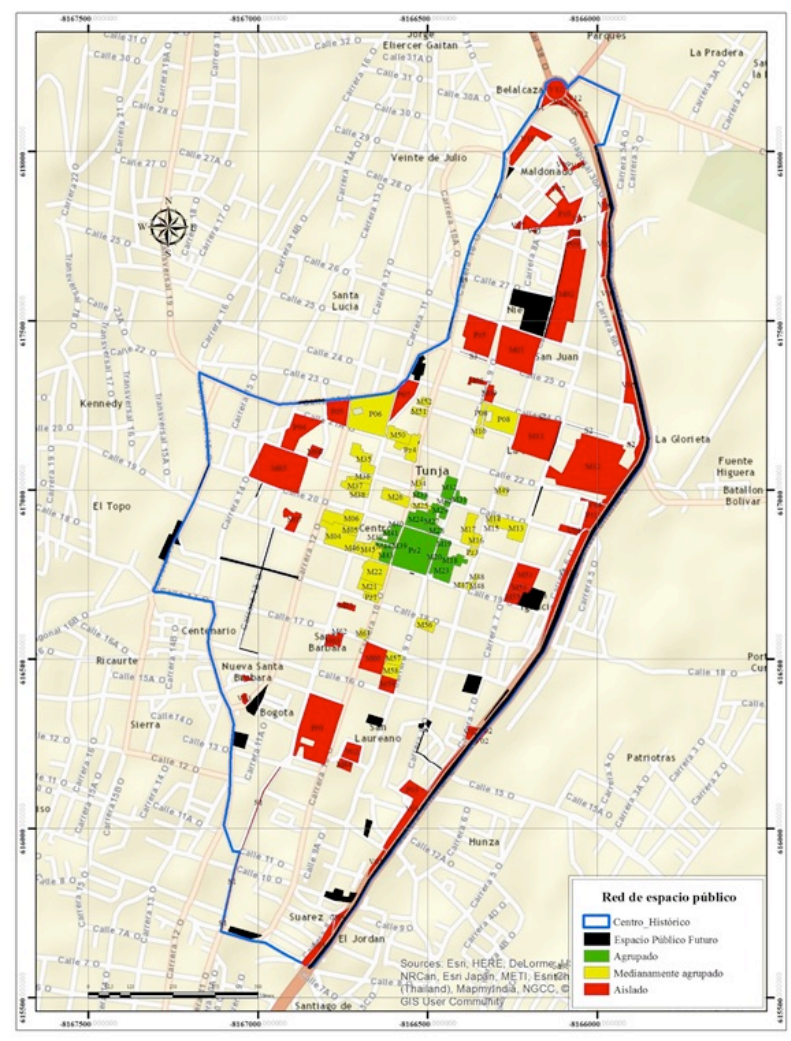

Figura 3. Lugares potenciales de generar espacio público verde. Fuente: Elaboración propia.

\section{Propuesta de recuperación y generación de espacio público}

\section{Recuperación de espacio público existente}

De acuerdo con las características de los sitios del listado de lugares con prioridad de recuperación, pertenecientes a la red de espacio público de la zona de conservación histórica de la ciudad de Tunja, se logra identificar que algunos lugares comparten características muy similares, por lo que se proponen ciertos tipos de estructuras de arquitectura viva para varios lugares a la vez, como es el caso de las casas-monumento, para las cuales se ha propuesto la instalación de jardines verticales; para parques y zonas verdes se ha propuesto la instalación de bioswales para su recuperación paisajística, además de generar una ayuda al sistema de drenaje de aguas lluvias de la zona. Estas intervenciones propuestas se detallan en la Tabla 4.

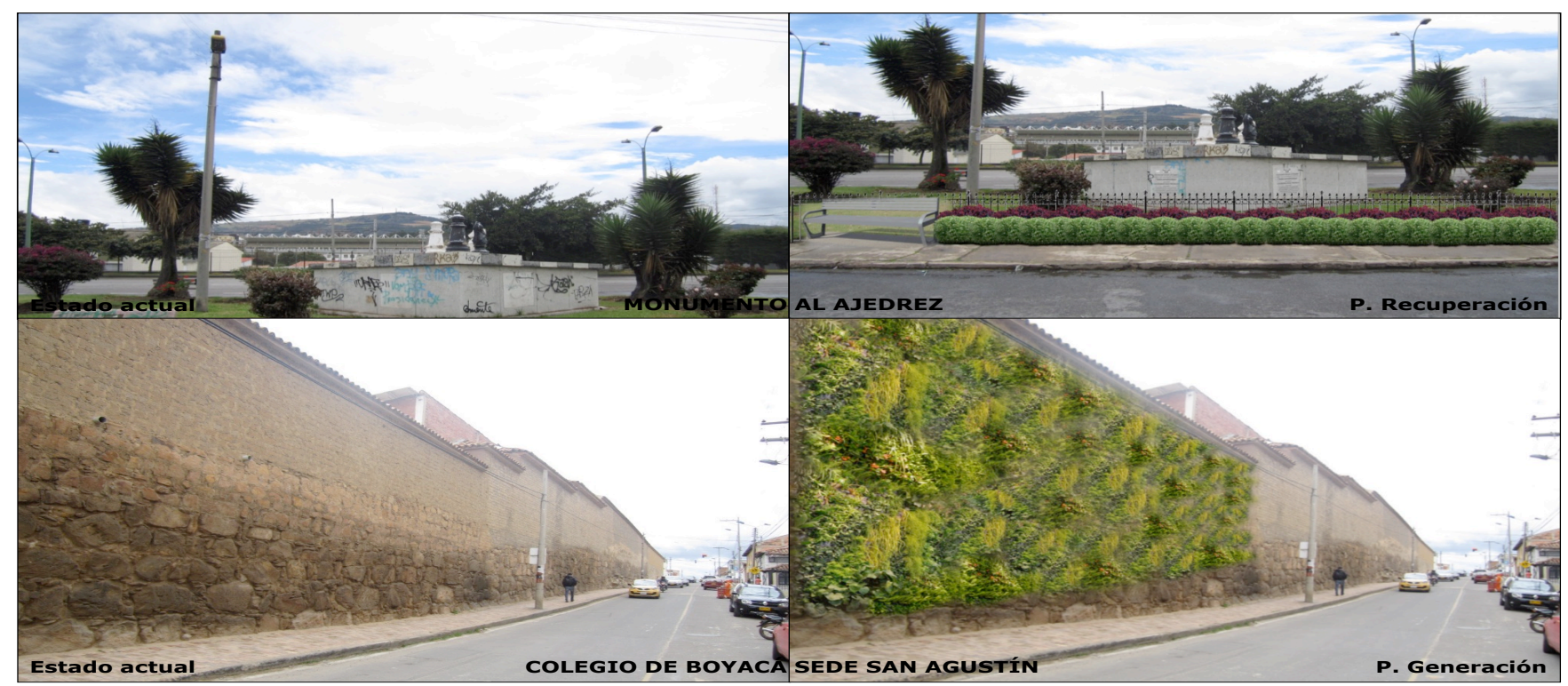

Figura 4. Recuperación del Monumento al ajedrez - Generación de espacio verde en el Colegio de Boyacá sede San Agustín. Fuente: Elaboración propia. 


\section{Nuevo espacio público verde}

En la Tabla 5 se detallan las estructuras de arquitectura viva asignadas a cada uno de los lugares listados donde figuran antejardines, amplios andenes, amplias fachadas de edificios, puentes peatonales y lotes baldíos principalmente, lugares para los que se logra proponer la ubicación de bioswales, jardines verticales, techos verdes y nuevos parques, para la generación de nuevos espacios verdes y el aumento de la cobertura vegetal.

Tabla 5

Propuesta de generación de espacio público verde

Lugar potencial

Descripción

Intersección Carrera 8 con Calle 15

Antejardín Iglesia Santuario Virgen del Topo

Andén Av. Maldonado con Diagonal 29

Antejardines Calle 22 entre Carrera 13 y Carrera 13a

Calle 18 entre Carrera 14 y Carrera12

Carrera 13 entre Calle 19 y Calle 17

Andén Calle 18 № 14-93

Talud vial Calle 14 con Carrera 8

Muro Ancianato Mi casita Hermanas de los Pobres

Muro Colegio ITECG

Muro Terminal de Transportes

Muro Universidad Antonio Nariño

Muro Colegio de Boyacá Sede San Agustín

Muro Cementerio Central

Muro Av. Maldonado № 25-39

Muro antiguo Colegio Castro Martínez

Mural Plazoleta San Francisco

Muro Alcaldía Municipio de Tunja

Avenida Oriental

Casa-lote Av. Colón № 16-32

Lote Carrera 12 № $14-30$

Lote Antiguo Seguro Social

Lote Calle 8 № 10-96

Lote Carrera 9 № $10-20$

Lote Calle 12 № 8-56
En esta intersección se encuentra un lote baldío en el que se propone la instalación de un parque; un amplio andén y un pequeño separador para los cuales se recomienda la instalación de bioswales.

Se propone embellecer con plantas florales y la instalación de bioswales contiguos a la vía de acceso al Santuario.

Se recomienda el embellecimiento de un amplio andén que, a pesar de poseer vegetación, no $\mathscr{\varrho}$ es considerado dentro de la red como un espacio verde por su estado físico decadente; $\frac{\pi}{3}$ entonces, se propone la instalación de un bioswale, convirtiéndose en un buen sumidero biológico en épocas de lluvias.

Embellecimiento con plantas florales.

Se aconseja aprovechar la amplitud de estos andenes para la instalación de sumideros biológicos, que irán ubicados en la parte exterior del andén y al nivel de la vía para que cumplan su función de drenaje.

Amplio andén al que se propone la instalación de un bioswale, que a su vez ayudaría en el drenaje de agua en épocas de lluvia.

Se propone la instalación de un ajardinamiento de fachada o muro vegetal, puesto que tiene una pendiente bastante pronunciada y cuenta con el acceso al sustrato para el crecimiento de las plantas.

Para todos estos muros se propone la instalación de muros vegetales, puesto que, para evitar daños en las paredes es necesario una estructura aislante en la que a su vez se soporten las plantas (Figura 4)
Se propone la instalación de una barrera vegetal en el separador de este corredor vial. La intervención se compone de un escaparate metálico o valla de altura media ubicada en el centro y a lo largo del separador, allí se soportará el crecimiento de plantas tipo trepadoras; cuando las plantas hayan crecido lo suficiente se obtendrá un muro verde en medio de las dos calzadas de esta avenida.

Para estos lotes, se propone la instalación de parques, en los que se incluyan jardines y el respectivo mobiliario urbano necesario.

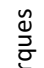

$\frac{\sigma}{\sigma}$ Este terreno presenta una pendiente elevada, sin embargo, en la parte más baja del lote existe una pequeña área considerablemente plana, por tanto, se propone la instalación de un parque en la parte baja y un ajardinamiento de fachada o muro vegetal en la parte alta o inclinada.

Para estos lotes, se propone la instalación de parques, en los que se incluyan jardines y el respectivo mobiliario urbano necesario. 


\begin{tabular}{|c|c|c|}
\hline Lote Calle 24 № $10-40$ & & \\
\hline Lote Calle 27 № 8-35 & & \\
\hline Lote Calle 27 № 8-55a & & \\
\hline Lote Calle 27 № 8-55b & & \\
\hline Puente peatonal Avenida Maldonado & 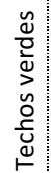 & $\begin{array}{l}\text { Los puentes de paso peatonal no son tenidos en cuenta como un espacio público importante } \\
\text { dentro de la red, sino considerados elementos inherentes a la vía pública en el proceso de } \\
\text { conformación de la ciudad. Sin embargo, dada su importancia en el desplazamiento peatonal, } \\
\text { se propone integrarlos a la red de espacio público como elementos de atractivo visual y } \\
\text { paisajístico mediante la instalación de un techo verde en cada uno de ellos. }\end{array}$ \\
\hline
\end{tabular}

Fuente: Elaboración propia.

\section{Discusión y conclusiones}

Mediante el diagnóstico de disponibilidad de espacio público en la zona de conservación histórica de la ciudad de Tunja, se observa que los lugares mejor amoblados son los parques, que cuentan con bancas, cestas de basura, elementos de comunicación, incluso casetas de venta, puesto que son necesarios varios elementos de mobiliario para soportar su multifuncionalidad (variedad de usos simultáneos que se pueden llevar a cabo en el lugar, entre los que están las actividades deportivas, la contemplación paisajística y actividades culturales).

Se establece que el estado general de las fachadas de las casas-monumento es Regular, dentro de lo que se registran estados de Bueno y Muy bueno, que coinciden con un uso institucional y/o comercial, y estados de Malo y Muy malo, relacionados con casos de abandono, siendo este tipo de lugar el que reporta más casos de abandono a pesar de su versatilidad (variedad de usos no simultáneos que se le pueden dar).

Esta situación puede estar influenciada por el hecho de que, aunque son catalogados como espacios públicos por el Plan de Ordenamiento Territorial de la ciudad, es necesario un proceso de compra o arrendamiento para darles un uso, institucional o comercial, usos que no son ciertamente públicos, pero que garantizan en cierta medida la conservación del mayor aporte de las casasmonumento a la red de espacio público, sus fachadas de apreciable arquitectura; entonces si no existe una institución o persona particular interesada en dar a estos inmuebles alguno de los usos mencionados, se presentan las situaciones de abandono y decadencia de las edificaciones ya mencionadas. Sin embargo, al hacer estos convenios público-privados se da un grado de privatización del espacio público que afecta las normas de convivencia democrática como la libertad de expresión (Salcedo, 2002).

La investigación permitió identificar los componentes clave de la red mediante el análisis de los tipos y frecuencias de usos de los lugares, en lo que las zonas verdes y separadores viales resultan ser conectores por ser lugares de paso, con tiempos de permanencia en ellos relativamente cortos o por ser parte del medio físico de acceso a otros lugares; los parques y monumentos son los nodos de la red, puesto que el viaje y desplazamiento hacia dichos lugares tiene un propósito específico con un tiempo de permanencia más largo; por último, las plazas y plazoletas principalmente son conectores, pero pueden llegar a comportarse como nodos, cuando ocasionalmente, son escenarios de actividades culturales y comerciales.

La condición paisajística y atractivo visual tiene los mejores puntajes en los espacios públicos verdes -zonas verdes, parques y separadores-, gracias a la abundante presencia vegetal, sin embargo, la vegetación está representada en su mayoría por especies arbóreas y césped, entonces, la ausencia de especies vegetales coloridas y variadas (no se encuentran plantas epífitas, acuáticas o trepadoras y hay pocas plantas florales) hace a estos lugares susceptibles de mejorar y ser más atractivos visualmente.

Los resultados de la valoración de la red, obtenidos a partir de la etapa de diagnóstico y soportados en las anteriores consideraciones, muestran que la totalidad de la red está valorada en 15,67 puntos (categoría Regular, cuyo valor se obtuvo del promedio de las valoraciones de todos los lugares de la red), resultado que a su vez confirma la necesidad percibida en los primeros análisis del diagnóstico, de proponer una alternativa de recuperación de la red de espacio público existente. 
Dentro de esta propuesta se destaca el uso de muros vegetales que realcen la belleza arquitectónica de las casas-monumento; la localización de coloridos jardines y bioswales; la ampliación de un parque y el uso de arcos vegetales (variación del muro vegetal) para el ornamento de las entradas de los parques y para la conformación de un túnel verde en la única ciclovía de la red de espacio público.

En síntesis, se logra visualizar que gran parte de los lugares de la red están ubicados en el centro de esta y, que por el contrario, a las afueras de la zona de estudio disminuye la cantidad de lugares presentes. Sin embargo, es el proceso de categorización de los lugares el que permite entender mejor la distribución espacial de estos dentro del sistema de espacio público y, a la vez, establecer que la configuración espacial de la red es en forma concéntrica, es decir, existe un centro espacial en el que se concentra gran cantidad de lugares públicos (único conjunto de lugares agrupados) a partir del cual, en dirección a los límites de la red, comienza a disminuir el número de espacios públicos disponibles, transición en la que se conforma un anillo de lugares medianamente agrupados y, finalmente, en la periferia se ubican los sitios más aislados del sistema. Así mismo, se establece que la red aún está interconectada en toda su extensión, con mayor medida en su centro y menor intensidad a las afueras, puesto que no se encuentra ningún lugar

\section{Referencias}

Alcântara Vasconcellos, E. (2010). Análisis de la movilidad urbana. Espacio, medio ambiente y equidad. Recuperado de http://www.caf.com/publicaciones

Bolund, P. \& Hunhammar, S. (1999). Ecosystem services in urban areas. Ecological Economics, 29(2), 293-301. https://doi.org/10.1016/s0921-8009(99)00013-0

Borja, J. y Muxí, Z. (2003). El espacio público, ciudad y ciudadanía. Barcelona: Electa.

Clark, M. \& Acomb, G. (2008). Bioswales-Vegetated swales. Recuperado de http://buildgreen.ufl.edu/Fact_sheet_bioswales_Veg etated Swales.pdf completamente incomunicado de la red con cero homólogos cercanos a $500 \mathrm{~m}$ de distancia.

La investigación permite plantear una alternativa de generación de nuevo espacio público verde que fortalezca la conectividad en la periferia de la red, proponiendo localidades, entre ellas lotes baldíos, muros, puentes peatonales, andenes y antejardines, cercanas a los lugares aislados de la red y con potencial de albergar la instalación de una estructura de arquitectura viva. Dentro de la propuesta de generación se destaca el aprovechamiento de los amplios andenes para la ubicación de canales de filtración biológica, la conversión de antejardines en amplios bioswales, la localización de techos verdes en las cubiertas de puentes peatonales, el uso de amplias fachadas para el emplazamiento de muros vegetales y la conversión de un separador vial en una barrera verde.

Finalmente, teniendo en cuenta que los principales resultados de la investigación se traducen en una propuesta de recuperación de espacio público existente y generación de nuevo espacio público verde, ambas mediante estructuras de arquitectura verde, se ratifica la hipótesis de que, al determinarse la disponibilidad del espacio público existente en el área urbana de la ciudad de Tunja, es posible la formulación de tipos específicos de infraestructuras verdes para su recuperación y generación $\mathbf{B}$

Departamento Administrativo Nacional de Estadística, DANE. (s/f). Estimación y proyección de población nacional, departamental y municipal total por área 1985-2020. Recuperado de https://www.dane.gov.co/index.php/estadisticaspor-tema/demografia-y-poblacion/proyecciones-depoblacion

Fernández-Cañero, R., Pérez, N., Quevedo, S., Pérez, L., y Franco, A. (2008). Ajardinamiento de fachadas y jardines verticales: otras formas de jardinería aplicadas a un desarrollo urbano más sostenible. Actas de Horticultura № 52. Innovación y futuro en la jardinería. I Simposio Iberoamericano- IV Jornadas Ibéricas de Horticultura Ornamental. Pontevedra, España. 
Forest Research (2010a). Benefits of green infrastructure. Report to Defra and CLG. Farnham, Surrey: Forest Research.

Forest Research (2010b). Benefits of green infrastructure. Report by Forest Research. Farnham, Surrey: Forest Research.

Ghimire, R., Ferreira, S., Green, G. T., Poudyal, N. C., Ken Cordell, H., \& Thapa, J. R. (2017). Green space and adult obesity in the United States. Ecological Economics, 136, 201-212.

https://doi.org/10.1016/j.ecolecon.2017.02.002h

Gómez, C. I. y Moyano, C. A. (2014). La calidad en el espacio público: en el eje ambiental y el parque de los periodistas. Revista Papeles de Coyuntura, 38, 66-80.

Lange Valdés, C. (2011). Dimensiones culturales de la movilidad urbana. Revista INVI, 26(71), 87-106. https://doi.org/10.4067/s0718-83582011000100004

Mayorga Mora, N. Experiencias de parques lineales en Brasil: espacios multifuncionales con potencial para brindar alternativas a problemas de drenaje y aguas urbanas / Natalia Mayorga Mora. p. cm. - (Nota técnica del BID; 518).

Medina Roa, J. (2009). Tunja, ciudad que emerge. Revista Credencial Historia. Edición 237.

Natural Resources Conservation Service. (2005). Bioswales... absorb and transport large runoff events. Recuperado de https://www.nrcs.usda.gov/Internet/FSE DOCUMEN TS/nrcs144p2 029251.pdf

Pascual González, A. y Peña Díaz, J. (2012). Espacios abiertos de uso público. Arquitectura y Urbanismo, 33(1), 25-42.

http://scielo.sld.cu/scielo.php?script=sci_arttext\&pid $=\mathrm{S} 1815-58982012000100003 \& \operatorname{lng}=e s \& \mathrm{t} \operatorname{lng}=\mathrm{es}$

Pickett, A., Cadenasso, M., Grove, J., Boone, C., Groffman, P., Irwin, E., Kaushal, S., ... \& Nilon, C. (2011). Urban ecological systems: Scientific foundations and a decade of progress. Journal of Environmental Management, 92(3), 331-362. https://doi.org/10.1016/j.jenvman.2010.08.022
Pinzón Botero, M. V., Torres, G. J., y Colonia Manzano, V. (2008). Bajo el convenio de Universidad Nacional de Colombia, Sede Palmira; Instituto de Estudios Ambientales - Idea y Alcaldía Municipal, Secretaría de Planeación Municipal. El espacio público como un sistema. Estatuto de Espacio Público Municipio de Palmira, p. 1-14.

Presidencia de la República de Colombia. (1998). Decreto 1504. (4, agosto, 1998). Por el cual se reglamenta el manejo del espacio público en los planes de ordenamiento territorial. Capítulo Primero Disposiciones Generales, Artículo $2^{\circ}$. Santa Fe de Bogotá D. C., p. 1-11.

Quintero González, L. E. (2017). Formulación de alternativas para recuperación de espacio público mediante infraestructuras verdes en Tunja (Tesis de grado inédita). Universidad Pedagógica y Tecnológica de Colombia, Sede Tunja, Colombia.

Ragheb, A., El-Shimy, H., \& Ragheb, G. (2016). Green architecture: A concept of sustainability. ProcediaSocial and Behavioral Sciences, 216, 778-787. https://doi.org/10.1016/j.sbspro.2015.12.075

Redondo Paredes, D. F. (2014). Beneficios socio ambientales de las infraestructuras verdes urbanas y su aplicación en la construcción y planificación urbanística en la Ciudad de Bucaramanga. PUENTE Revista Científica, 8(2), 15-23. Recuperado de https://revistas.upb.edu.co/index.php/puente/article /view/7128

Rolfsdotter-Jansson, C. (2009). Ekostaden Augustenborg on the way towards a sustainable neighborhood. Recuperado de

http://climate-adapt.eea.europa.eu/metadata/casestudies/urban-storm-water-management-inaugustenborg-malmo/augustenborg-brochure.pdf

Roy, M. (2008). Importance of green architecture today. Green Architecture: A concept of sustainability. Procedia-Social and Behavioral Sciences, 216, 778787.

Salcedo, R. (2002). El espacio público en el debate actual: una reflexión crítica sobre el urbanismo postmoderno. Revista EURE, 28(84). https://doi.org/10.4067/s0250-71612002008400001 
Suárez, A., Camarena, P., Herrera, I., y Lot, A. (2011). Infraestructura verde y corredores ecológicos de los pedregales: ecología urbana del sur de la Ciudad de México (1ำ edición). México, DF: Universidad Nacional Autónoma de México.

U.S. Green Building Council, USGBC. (2002). National trends and prospects for high-performance green buildings. Recuperado de https://www.usgbc.org/resources/buildingmomentum-national-trends-and-prospects-highperformance-green-buildings

U.S. Environmental Protection Agency, EPA, Office of Wastewater Management. (2015). Tools, strategies and lessons learned from EPA Green Infrastructure Technical Assistance Projects. Washington, D.C.: EPA.
Vandermeulen, V., Verspecht, A., Vermeire, B., Van Huylenbroeck, G., \& Gellynck, X. (2011). The use of economic valuation to create public support for green infrastructure investments in urban areas. Landscape and Urban Planning, 103(2), 198-206.

https://doi.org/10.1016/j.landurbplan.2011.07.010

Vásquez, E. (2016). Infraestructura verde, servicios ecosistémicos y sus aportes para enfrentar el cambio climático en ciudades: el caso del corredor ribereño del río Mapocho en Santiago de Chile. Revista de Geografía Norte Grande, 63, 63-86.

https://doi.org/10.4067/s0718-34022016000100005 